\title{
UVES/VLT high resolution absorption spectroscopy of the GRB 080330 afterglow: a study of the GRB host galaxy and intervening absorbers ${ }^{\star}$
}

\author{
V. D’Elia ${ }^{1}$, F. Fiore ${ }^{1}$, R. Perna ${ }^{2}$, Y. Krongold ${ }^{3}$, S. D. Vergani ${ }^{4}$, S. Campana ${ }^{5}$, S. Covino ${ }^{5}$, P. D’Avanzo ${ }^{5}$, D. Fugazza ${ }^{5}$, \\ P. Goldoni ${ }^{6,7}$, C. Guidorzi ${ }^{5}$, E. J. A. Meurs ${ }^{8,9}$, L. Norci ${ }^{9}$, S. Piranomonte ${ }^{1}$, G. Tagliaferri ${ }^{5}$, and P. Ward ${ }^{10}$ \\ INAF - Osservatorio Astronomico di Roma, via Frascati 33, 00044 Monteporzio Catone, Italy \\ e-mail: delia@mporzio.astro.it \\ 2 JILA, Campus Box 440, University of Colorado, Boulder, CO 80309-0440, USA \\ 3 Instituto de Astronomia, Universidad Nacional Autonomica de Mexico, Apartado Postal 70-264, 04510 Mexico DF, Mexico \\ 4 University Paris 7, APC, Lab. Astroparticule et Cosmologie, UMR7164 CNRS, 10 rue Alice Domon et Lonie Duquet, \\ 75205 Paris Cedex 13, France \\ 5 INAF, Osservatorio Astronomico di Brera, via E. Bianchi 46, 23807 Merate (LC), Italy \\ 6 Laboratoire Astroparticule et Cosmologie, 10 rue A. Domon et L. Duquet, 75205 Paris Cedex 13, France \\ 7 Service d'Astrophysique, DSM/DAPNIA/SAp, CEA-Saclay, 91191 Gif-sur-Yvette, France \\ 8 School of Cosmic Physics, DIAS, 31 Fitzwilliam Place, Dublin 4, Ireland \\ 9 School of Physical Sciences and NCPST, DCU, Glasnevin, Dublin 9, Ireland \\ 10 Dunsink Observatory, Castleknock, Dublin 15, Ireland
}

Received 16 January 2009 / Accepted 15 May 2009

\begin{abstract}
Aims. We study the gamma-ray burst (GRB) environment and intervening absorbers by analyzing the optical absorption features produced by gas surrounding the GRB or along its line of sight.

Methods. We analyzed high resolution spectroscopic observations $(R=40000, S / N=3-6)$ of the optical afterglow of GRB 080330 , taken with UVES at the VLT $\sim 1.5 \mathrm{~h}$ after the GRB trigger.

Results. The spectrum illustrates the complexity of the ISM of the GRB host galaxy at $z=1.51$ which has at least four components in the main absorption system. We detect strong FeII, SiII, and NiII excited absorption lines associated with the bluemost component only. In addition to the host galaxy, at least two more absorbers lying along the line of sight to the afterglow have been detected in the redshift range $0.8<z<1.1$, each exhibiting MgII absorption. For the bluemost component in the host galaxy, we derive information about its distance from the site of the GRB explosion. We do so by assuming that the excited absorption lines are produced by indirect UV pumping, and compare the data with a time dependent photo-excitation code. The distance of this component is found to be $\sim 280_{-50}^{+40} \mathrm{pc}$, which is lower than found for other GRBs (1-6 kpc). We identify two additional MgII absorbers, one of them with a rest frame equivalent width larger than $1 \AA$.

Conclusions. The distance between the GRB and the absorber measured in this paper confirms that the power of the GRB radiation can influence the conditions of the interstellar medium up to a distance of at least several hundred pc.

For the intervening absorbers, we confirm the trend that on average one strong intervening system is found per afterglow, as has been noted in studies exhibiting an excess of strong MgII absorbers along GRB sightlines compared to quasars.
\end{abstract}

Key words. gamma rays: bursts - cosmology: observations - galaxies: abundances - ISM: general

\section{Introduction}

The study of the interstellar medium (ISM) of $z \gtrsim 1$ galaxies has so far relied upon observation of Lyman-break galaxies (LBGs) at $z=3-4$ (see e.g. Steidel et al. 1999) and galaxies that happen to be along lines of sight to bright background quasars (or QSOs, see e.g., Prochaska et al. 2003). However, LBGs are characterized by significant star formation and their inferred chemical abundances may not be representative of typical high- $z$ galaxies. Weak metal-line systems along the line of sight to quasars probe mainly galaxy haloes, rather than their bulges or discs. Taking advantage of ultra-deep Gemini multi-object spectrograph

* Based on observations collected at the European Southern Observatory, ESO, the VLT/Kueyen telescope, Paranal, Chile, in the framework of programs 080.A-0398. observations, Savaglio et al. $(2004,2005)$ studied the ISM of a sample of faint $K$-band selected galaxies at $1.4<z<2.0$, finding MgII and FeII abundances much higher than in QSO systems but similar to those in GRB host galaxies (Prochaska et al. 2007). These studies can hardly be extended to higher redshift with the present generation of $8 \mathrm{~m}$ class telescopes, because of the faintness of high- $z$ galaxies. Since the discovery that gammaray bursts (GRBs) are extragalactic, we are now able to use an independent tool to study the ISM of high- $z$ galaxies.

The main difference between QSO and GRB absorption spectroscopy is that QSOs are stationary in their emission, while GRBs are the most variable and violent phenomena in the Universe known to date. Thus, while the QSO has already ionized the ISM along its line of sight, the physical, dynamical, and chemical status of the circumburst medium in the star-forming 
region hosting the GRB progenitor can be modified by the explosive event, by shocks and ionizing photons. The presence of blue-shifted absorbers may indicate that the GRB and its afterglow can not only ionize the interstellar medium, but further radiatively and/or collisionally accelerate it. Circumstellar absorption lines in GRB afterglow spectra can be used to determine the main properties of the stellar progenitors (van Marle et al. 2005). The variability in the intensity of absorption lines can be used to determine the location and density of the absorber, following Perna \& Loeb (1998), Boettcher et al. (2002), Draine \& Hao (2002), Mirabal et al. (2002), Perna \& Lazzati (2002), and Perna et al. (2003).

Occasionally, extremely bright optical transient emission is associated with a GRB event, offering a superb opportunity to investigate high- $z$ galaxies by means of high resolution spectroscopy of the optical transient.

Fiore et al. (2005) presented the first high-resolution spectroscopic data of GRB afterglows. Their study of the UVES/VLT spectra of GRB 020813 and GRB 021004 showed that the ISM of GRB host galaxies is complex when resolved down to a width of a few tens of $\mathrm{km} \mathrm{s}^{-1}$, many velocity components contributing to each main absorption system and spanning a total velocity range of up to thousands of $\mathrm{km} \mathrm{s}^{-1}$.

Since the launch of Swift, which now allows a prompt reaction to a GRB event, a dozen afterglows have been observed at high resolution. The first published high resolution observations in the Swift era were those of the GRB 051111 and GRB 050730 afterglows (Chen et al. 2005; Prochaska et al. 2006; Penprase et al. 2006). They confirm the complex nature of the ISM of the GRB host galaxies, and velocity ranges of hundreds of $\mathrm{km} \mathrm{s}^{-1}$ are found with the spectra obtained with MIKE at the MagellanII and HIRES at the Keck. GRB 050730 was also observed using UVES at the VLT (D'Elia et al. 2007): the ISM is inferred to have a clumpy nature from the presence of six components in the high resolution spectrum, each representing a different layer of absorbing gas. The capability of resolving the absorbing gas into components of different velocity allows us to separate the contribution of the circumburst environment from that of regions in the host galaxy far away and less affected by the GRB afterglow, as in the case of GRB 050922C (Piranomonte et al. 2008). Fine structure and other excited levels of the species OI and the ions FeII, NiII, SiII, and CII, are routinely identified in GRB spectra. These lines are most probably excited by the intense UV flux coming from the afterglow, since strong variation is observed when multi-epoch, high resolution spectroscopy is available. This variation is inconsistent with a pure infrared excitation or collisional processes (Vreeswijk et al. 2007; D'Elia et al. 2009). Thus, by assuming UV pumping as the responsible mechanism for the production of these lines, we can compute the distance of the gas from the GRB. This distance is found to be of the order of a few kpc (see Vreeswijk et al. 2007, for GRB 060418) or even larger, as in the case of the naked-eye GRB 080319B (D'Elia et al. 2009), for which the inferred distance of $6 \mathrm{kpc}$ to the farthermost component allows speculation about a possible extragalactic origin of part of the excited gas.

GRB high resolution spectroscopy is also suitable for studying the systems lying along the line of sight to GRBs, and not only their host galaxies. Surprisingly, the number density of MgII intervening absorbers in GRB spectra is nearly 4 times higher than that along QSO sightlines (Prochter et al. 2006), while the CIV absorbers do not show any statistical difference (Sudilovsky et al. 2007; Tejos et al. 2007). The reason for the MgII excess in GRB spectra is still uncertain, and a larger sample is required to properly address this issue.
In this paper, we discuss high resolution spectroscopy of GRB 080330. We investigate both the local medium surrounding the GRB, and the intervening systems. For what concerns the host galaxy, we focus in particular on the analysis of the absorption lines produced by the excited levels of the atoms and ions constituting the absorbing gas. The comparison of these absorption features and their corresponding ground states yields information about the gas distance from the GRB explosion site, based on the assumption of indirect UV pumping production of the excited levels. A full analysis of the intervening absorbers lying along the line of sight to the GRB afterglow is also presented.

The paper is organized as follows. Section 2 summarizes of the GRB 080330 detection and observations. Section 3 presents the high resolution observations and data reduction. Section 4 is devoted to the study of the absorbers located in the host galaxy, and in particular to the analysis of their excited levels. Section 5 presents a full analysis of the absorbing systems identified in the spectrum. Finally, in Sect. 6 the results are discussed and conclusions are drawn.

\section{GRB 080330}

GRB 080330 was discovered by Swift-BAT on March 30, 2008, at 03:41:16 UT (Mao et al. 2008). Less than $100 \mathrm{~s}$ later, both XRT and UVOT detected the afterglow, which had a magnitude of 18.8 (white filter). Even before the distribution of the XRT position, the TAROT and ROTSE robotic telescopes had detected the optical afterglow at the position RA: 11:17:04.5 Dec: $+30: 37: 24.0$ (J2000). The magnitude of the object was roughly $17.522 \mathrm{~s}$ after the trigger, and reached a peak of $R=16.8$ at 300 s (Klotz et al. 2008; Schaefer \& Guver 2008). The redshift of the host galaxy was first secured by NOT observations (Malesani et al. 2008), and then confirmed by Cucchiara et al. (2008), using Hobby-Eberly Telescope observations. The afterglow was also detected in the infrared band $(J, H$, and $K$ ) by PAIRITEL (Bloom \& Starr 2008) and GROND (Clemens et al. 2008), and in the $\gamma$-band $(20 \mathrm{keV}-1 \mathrm{MeV})$ by Konus-Wind (Golenetskii et al. 2008). For a detailed analysis of the multi-band properties of the prompt and afterglow emission evolution, we refer the reader to Guidorzi et al. (2009).

\section{Observations and data reduction}

In the framework of the ESO program 080.A-0398, we observed the afterglow of GRB 080330 with the high resolution UV-visual echelle spectrograph (UVES, Dekker et al. 2000), mounted at the VLT-UT2 telescope. Observations began on March 30, 2008 at 05:06:32 UT $(\sim 1.5 \mathrm{~h}$ after the GRB) for a net exposure time of $45 \mathrm{~min}$, when the magnitude of the afterglow was $R \sim 18.8$. Because of observational and scheduling constraints, only the UVES dichroic 1 was used, when employing the red as well as the blue arm. The slit width was set to be $1^{\prime \prime}$ (which corresponds to a resolution of $R=40000$ ) and the read-out mode was rebinned to $2 \times 2$ pixels. The achieved spectral range covers the bands from $\sim 3300 \AA$ to $\sim 3870 \AA, \sim 4780 \AA$ to $\sim 5750 \AA$, and $\sim 5830 \AA$ to $\sim 6810 \AA$. The data below $\sim 3500 \AA$ is dominated totally by noise. Table 1 provides a summary of the UVES setups used.

The data reduction was performed using the UVES pipeline (Ballester et al. 2000). The final resolution was $10.5 \mathrm{~km} \mathrm{~s}^{-1}$ for the blue arm and $7.5 \mathrm{~km} \mathrm{~s}^{-1}$ for the red arm; this slight loss 
Table 1. UVES setups.

\begin{tabular}{lcccc}
\hline \hline Setup $(\mathrm{nm})$ & Wavelength $(\AA)$ & Slit width & Spec. Resolution & $S / N$ \\
\hline Dic 1, 346 & $3300-3870$ & $1^{\prime \prime}$ & 40000 & $\sim 3$ \\
Dic 1, 580 & $4780-6810$ & $1^{\prime \prime}$ & 40000 & $\sim 6$ \\
\hline
\end{tabular}

Table 2. Absorption line column densities for component IV of the main system.

\begin{tabular}{|c|c|c|}
\hline Species & Observed transitions & $\overline{\log N\left(\mathrm{~cm}^{-2}\right)}$ \\
\hline $\mathrm{SiII}{ }^{2} \mathrm{P}_{1 / 2}^{\circ}$ & $\lambda 1526$ & $15.41 \pm 0.22(S)$ \\
\hline $\mathrm{SiIII}^{2} \mathrm{P}_{3 / 2}^{\circ}$ & $\lambda 1533$ & $14.25 \pm 0.11$ \\
\hline $\operatorname{MgI}{ }^{1} \mathrm{~S}_{0}$ & $\lambda 2026$ & $13.51 \pm 0.08$ \\
\hline $\mathrm{ZnII}{ }^{2} \mathrm{~S}_{1 / 2}$ & $\lambda 2026, \lambda 2062$ & $12.79 \pm 0.06$ \\
\hline CrII a ${ }^{6} S_{5 / 2}$ & $\lambda 2056, \lambda 2062, \lambda 2066$ & $13.62 \pm 0.04$ \\
\hline NiII ${ }^{4} \mathrm{~F}_{9 / 2}$ & $\lambda 2166, \lambda 2217, \lambda 2223$ & $13.07 \pm 0.04$ \\
\hline $\mathrm{MnII} \mathrm{a}^{7} \mathrm{~S}_{3}$ & $\lambda 2576, \lambda 2594, \lambda 2606$ & $13.13 \pm 0.03$ \\
\hline FeII a ${ }^{6} D_{9 / 2}$ & $\begin{array}{c}\lambda 2249, \lambda 2260, \lambda 2344, \\
\lambda 2374, \lambda 2382, \lambda 2586, \\
\lambda 2600\end{array}$ & $14.70 \pm 0.09$ \\
\hline FeII a ${ }^{6} D_{7 / 2}$ & $\begin{array}{c}\lambda 2333, \lambda 2365, \lambda 2389, \\
\lambda 2396, \lambda 2599, \lambda 2612, \\
\lambda 2626\end{array}$ & $13.76 \pm 0.03$ \\
\hline FeII $a^{6} D_{5 / 2}$ & $\begin{array}{c}\lambda 2328, \lambda 2349, \lambda 2399, \\
\lambda 2405, \lambda 2607, \lambda 2618, \\
\lambda 2632\end{array}$ & $13.46 \pm 0.04$ \\
\hline FeII $a^{6} D_{3 / 2}$ & $\begin{array}{c}\lambda 2359, \lambda 2407, \lambda 2411, \\
\lambda 2614, \lambda 2631\end{array}$ & $13.32 \pm 0.04$ \\
\hline FeII $a^{6} D_{1 / 2}$ & $\begin{array}{c}\lambda 2411, \lambda 2414, \lambda 2622, \\
\lambda 2629\end{array}$ & $12.93 \pm 0.05$ \\
\hline FeII a ${ }^{4} F_{9 / 2}$ & $\lambda 2332, \lambda 2348, \lambda 2360$ & $14.04 \pm 0.05$ \\
\hline
\end{tabular}

of resolution in the blue band is caused by a rebinning to increase the signal-to-noise ratio per pixel, which ranges from $\sim 3$ at $3800 \AA$ to $\sim 6$ at $6800 \AA$. The noise spectrum, used to determine the errors in the best-fit line parameters, was calculated from the real, background-subtracted spectrum, using line-free regions. This, therefore, takes into account both statistical and systematic errors in the pipeline processing as well as the background subtraction.

In the following two sections, we discuss the absorption features related to the main system, i.e., the host galaxy (Sect. 4), and those produced by other intervening systems (Sect. 5).

\section{The main absorption system}

This system has the greatest number of features and is produced by the gas residing in the GRB host galaxy. The spectrum exhibits a large number of metal-absorption lines, of both neutral (MgI) and low ionization species (SiII, ZnII, CrII, NiII, FeII, MnII). In addition, strong absorption from the fine structure levels of FeII and SiII and from the metastable levels of the FeII and NiII has been identified, suggesting that the intense radiation field from the GRB excites these features. Table 2 summarizes all the absorption lines of the main system. The ISM of the host galaxy is complex, with many components contributing to the absorption system. The wealth of metal-line transitions allows us to precisely determine the redshift of the GRB host galaxy. This yields a heliocentric redshift of $z=1.5115$, if we set the reference point to the bluemost component of the main system; this component is the only one for which there is significant absorption from the excited levels of the intervening gas (see Sect. 4.2). Our redshift value agrees with that found by Malesani et al. (2008) and Cucchiara et al. (2008). This complex environment is described in the next following sections with a detailed study of the excited lines that aims to estimate the distance of the circumburst gas.

\subsection{Line-fitting procedure}

The analysis of the GRB environment is complicated by the complexity of the absorption lines in the spectrum, which in several cases cannot easily be fitted with a single line profile. This implies that many different velocity components contribute to the gas in the GRB environment. In other words, several layers of gas that may be close to or far from each other, appear to be mixed together in the spectrum (in velocity space). The presence of several components is thus indicative of clumpy gas in the GRB environment, which consists of different absorbing regions each with different physical properties.

If the absorption features are fitted with a single Voigt profile, we are able to obtain only very poor fitting parameters and very high $\chi^{2}$ values. The identification of the different components is somewhat subjective, the true message being that the geometry and kinematics of the ISM clouds probed by the GRB line of sight are complex. The FeII lines have the widest velocity range, so we tried to use them to identify the different components within the circumburst matter. Although several FeII absorption features are saturated, two of them ( $\lambda 2260$ and $\lambda 2249)$ are not and can guide the fit in identifying the wavelengths of the components. A four-component model represents a good fit for the FeII lines. Thus, the redshifts of these components as well as the Doppler parameters were fixed to fit all the saturated FeII and other ion features present in the spectrum with the same model.

Figure 1 presents all the absorption features relative to FeII, with the exception of the saturated lines. The transitions due to the four fine structure levels of the FeII ground state are marked with asterisks. The lines FeII $\lambda 2332$ and $\lambda 2360$ are produced by the first excited level. The components are labeled I (redmost) to IV. The reference zeropoint of the velocity shifts has been placed at $z=1.5115$. This value was chosen to coincide with the redshift of the bluemost component, for two reasons. First, the narrow profile of the excited lines makes the redshift determination more accurate. Second, the excited levels are possibly produced by the indirect UV-pumping mechanism (see next subsection), implying that component IV is probably the closest component to the GRB explosion site. Figure 2 displays all the other species observed in the spectrum, namely: MgI, ZnII, CrII, SiII, MnII, and NiII. The transition SiII $\lambda 1533^{*}$ is produced by the first fine structure level of the SiII, and all the observed NiII lines belong to the first excited level. We note that all the excited absorption features in the spectrum, produced by fine structure and/or metastable levels of FeII, SiII, and NiII, show a significant absorption only in component IV, i.e., our bluemost, reference component (the NiII metastable level features also a faint absorption in component III). Table 2 lists all the transitions observed in the spectrum for each species. The column densities for all the elements and ions of the host galaxy absorbing gas, estimated using this four component model, are also reported. The Table shows only the column densities of component IV, which is the only one used in the following analysis, due to the presence of the excited transitions. We emphasize here that the SiII $\lambda 1526$ line is strongly saturated, thus the corresponding column density in Table 2, marked with an "S", should be interpreted with care. 

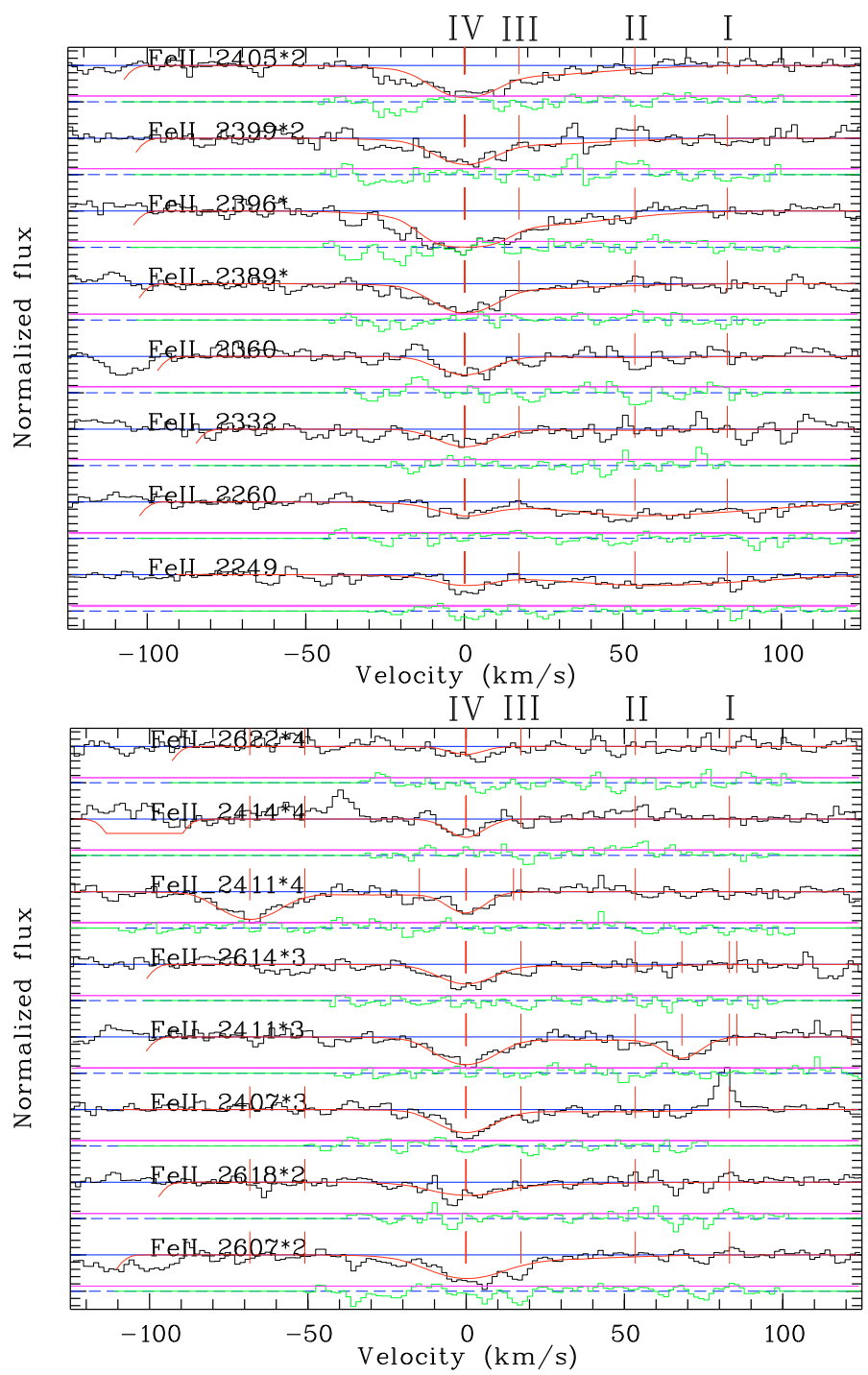

Fig. 1. The FeII ground and excited absorption features. Solid lines represent our four Voigt components, best-fit model. Vertical lines identify the velocity of each component with respect to the zeropoint arbitrarily placed at the redshift of the bluemost component $(z=1.5115)$. Bottom, solid lines mark the fit residuals.

As described in the introduction, the observation of excited states in GRB absorption spectra is a quite common feature. The next sub-section is thus devoted to these features and to the information that can be extracted from their analysis.

\subsection{Excited levels}

The level structure of an atom or ion is characterized by a principal quantum number $n$, which defines the atomic levels, and by the spin-orbit coupling (described by the quantum number $j$ ), which divides these levels into fine structrure sublevels. In GRB absorption spectra, several excited features are detected at the GRB redshift, because of the population of both $n>1$ and/or $n=1$ fine structure levels. As mentioned before, component IV of the main system in the spectrum of GRB 080330 shows absorption from the four fine structure levels of the FeII ground state $\left(\mathrm{a}^{6} \mathrm{D}\right)$ and from the first sublevel of the $n=2$ quantum state $\left(a^{4} F_{9 / 2}\right)$. Absorption from the SiII ${ }^{2} \mathrm{P}_{3 / 2}^{\circ}$ fine structure level and

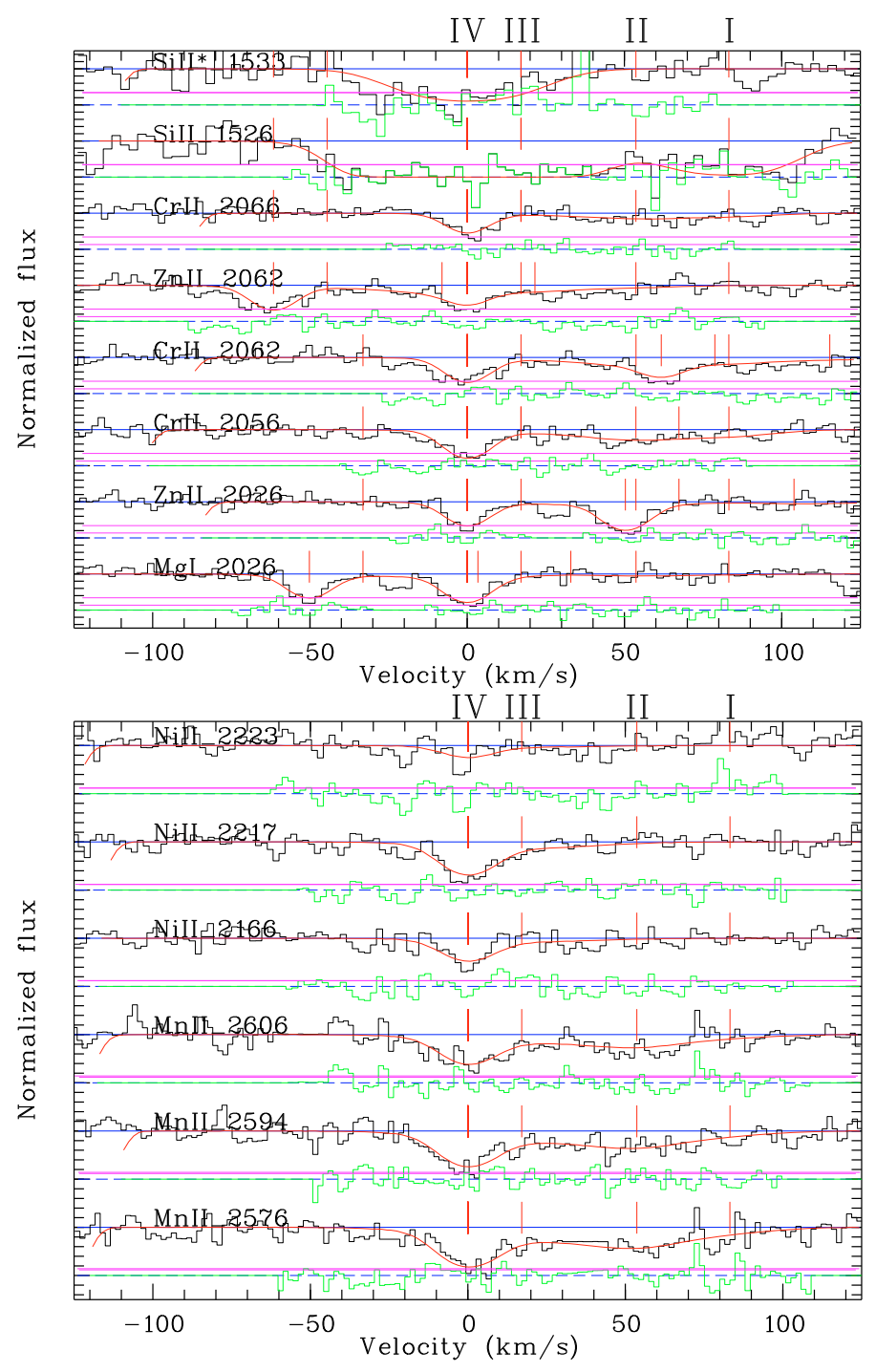

Fig. 2. The MgI, ZnII, CrII, SiII (top panel), MnII, and NiII (bottom panel) absorption features. Solid lines represent our best-fit model. Vertical lines identify the velocity of each component with respect to the zero point arbitrarily placed at the redshift of the bluemost component $(z=1.5115)$. Bottom, solid lines mark the fit residuals.

from the NiII ${ }^{4} \mathrm{~F}_{9 / 2}$ metastable level are also present (see Table 2 for details).

There are basically two mechanisms for exciting the gas of the GRB host galaxy to these states. The first is by collisional effects (if the density is sufficiently high, i.e., $\geq 10^{5} \mathrm{~cm}^{-3}$ ), and the second is by the absorption of electromagnetic radiation. In the latter case, the absorbed radiation can be from the infrared band, by a direct population of the fine structure levels of the ground state and other excited levels with a low value of $n$, or from the UV band, by the population of higher levels followed by the depopulation into the states responsible for the absorption features. Multi-epoch high resolution spectroscopy plus the accurate modeling of the atomic level population has proven to be a powerful tool for discriminating between these two processes. The strong variability in the column density of the FeII and NiII excited levels observed in GRB 060418 (Vreeswijk et al. 2007) and GRB 080319B (D'Elia et al. 2009) excluded collisional processes and direct infrared pumping as being responsible for the excitation. Since we do not have multi-epoch spectroscopic data, 
we cannot repeat the same procedure for this GRB, but must assume that UV pumping is at work.

By assuming indirect UV pumping as the dominant mechanism for producing the excited levels also in component IV of GRB 080330, we can compare our observed column densities to those predicted by a photoexcitation code for the time when the high resolution observations were acquired. This can be completed for the FeII and SiII ions, for which both ground states and excited levels are present in the spectrum. In contrast, for NiII, we only measured the column density of the first metastable level, since the transition corresponding to the ground level fell in the gap between the blue and the red regions of the spectrum. The photoexcitation code is that used by D'Elia et al. (2009). Basically, it solves the detailed balance equation

$\frac{\mathrm{d} N_{\mathrm{u}}}{\mathrm{d} t}=N_{\mathrm{l}} B_{\mathrm{lu}} F_{v}\left(\tau_{0}\right)-N_{\mathrm{u}}\left[A_{\mathrm{ul}}+B_{\mathrm{ul}} F_{v}\left(\tau_{0}\right)\right]$,

in a time-dependent way for all the transitions involving the 16 lowest levels plus 22 higher excited states (for FeII) and the 20 lowest levels (for SiII). The emission terms in that equation depend on the flux level experienced by the absorbing gas. This flux is of course a function of the distance of the gas from the GRB explosion site, which is a free parameter of the computation. The code takes into account radiative transfer and possible opacities of some of the transitions. The other free parameters are the initial column density of the ion, which is assumed to be in the ground state before the GRB flux reaches it, and the Doppler parameter of the gas. The flux of the GRB as a function of time was measured by Guidorzi et al. (2009). Following the notation

$F_{R}(t)=\frac{F_{R}\left(t_{0}\right)\left(t / t_{0}\right)^{-\alpha}\left(v / v_{R}\right)^{-\beta}}{(1+z)}$

$F_{R}(t)$ is the flux in the $R$ band at the time $t$, and the normalization of the flux is $F_{R}\left(t_{0}\right)=4.64 \times 10^{-27} \mathrm{erg} \mathrm{cm}^{-2} \mathrm{~s}^{-1}$ at the time $t_{0}=317 \mathrm{~s}$. The temporal index is $\alpha=-0.56$ before $t_{0}, \alpha=$ 0.15 between $t_{0}$ and $t_{1}=1456 \mathrm{~s}$ and $\alpha=1.08$ for $t>t_{1}, v_{R}$ is the central frequency of the $R$ band. We assume here a spectral index of $\beta=0.8$ (Guidorzi et al. 2009); this value is close to the value of $\beta=0.7$ inferred using the multispectral data from Cobb (2008). The flux experienced by the absorber is

$F_{v}^{\mathrm{ABS}}(t)=\left(d_{L, \mathrm{GRB}} / d\right)^{2} \times F_{v}(t)$,

where the GRB luminosity distance $d_{L, \text { GRB }}$ was computed assuming an $H_{0}=70 \mathrm{~km} \mathrm{~s}^{-1} \mathrm{Mpc}^{-1}, \Omega_{\mathrm{M}}=0.3, \Omega_{\Lambda}=0.7 \mathrm{cos}-$ mology. $F_{v}^{\mathrm{ABS}}(t)$ was corrected by the radiative transfer theory, before using it in Eq. (1). The initial column densities of the ground states of FeII and SiII were computed from the observed column densities of all the levels of each ion. The exact values are: $N_{\text {FeII }}=14.88 \pm 0.15$ and $N_{\text {SiII }}=15.44 \pm 0.25$. Finally, the Doppler parameter was allowed to vary between 10 and $20 \mathrm{~km} \mathrm{~s}^{-1}$, i.e., the values that reproduce most closely the excited lines alone and all the absorption features together, respectively. Of course all these values refer solely to component IV, the only one for which the excited levels are present in the spectrum. All the details of this time-dependent photo-excitation code can be found in D'Elia et al. (2009).

We first modeled the FeII photo-excitation. The value of the Doppler parameter that provides the closest agreement is $b=10 \mathrm{~km} \mathrm{~s}^{-1}$. Figure 3 shows the model that reproduces the FeII data most closely. The $x$-axis is the rest-frame time interval between the GRB explosion and the UVES observation. The time of observation (with respect to the GRB detection) is defined as the logarithmic mean between the beginning and the

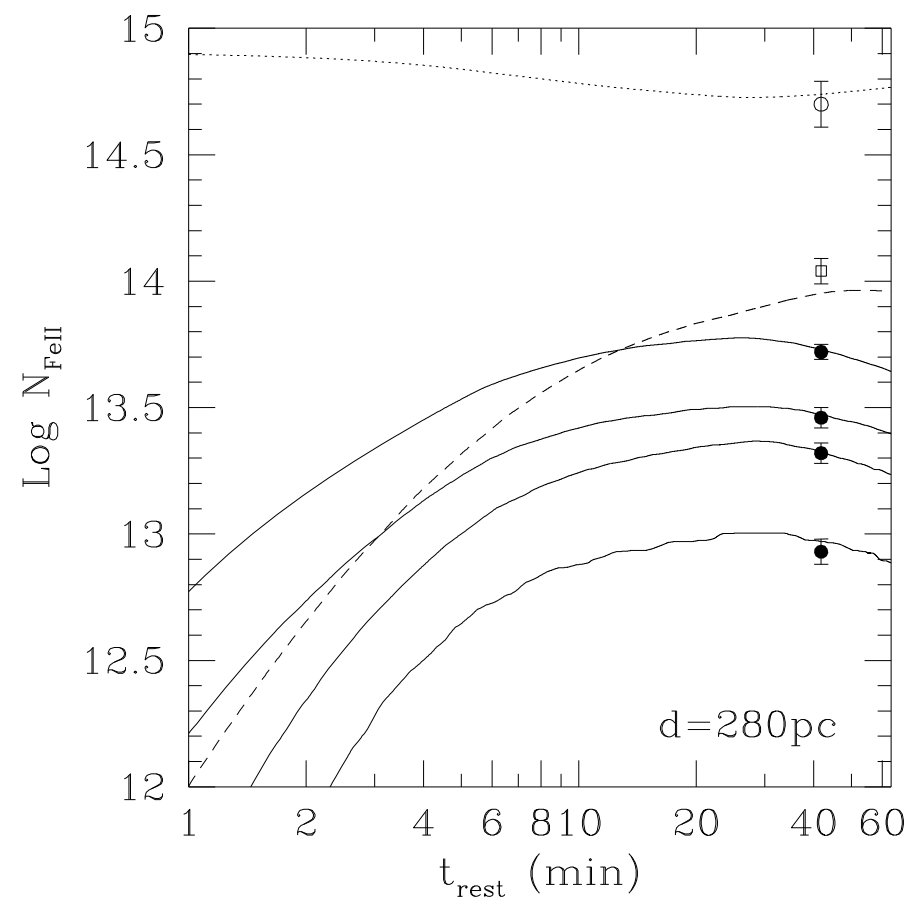

Fig. 3. FeII column densities for the ground level (open circle), fine structure level (solid circles), and first excited level (square) transitions for component IV in the spectrum of GRB 080330. Column density predictions from our time-dependent photo-excitation code are also shown. They refer to the ground level (dotted line), fine structure levels (solid lines), and first excited level (dashed line) transitions, in the case of an absorber placed at $280 \mathrm{pc}$ from the GRB.

end of the observation. The agreement between the theoretical curves and the data is very good. The distance of component IV from the GRB explosion site is measured to be $d=280 \mathrm{pc}$. To estimate the error associated to $d$, we ran our code for a grid of distances in the range $50-800 \mathrm{pc}$. We then converted the column densities and the corresponding errors to linear values to compute the $\tilde{\chi}^{2}$. Figure 4 plots this quantity as a function of $d$, which has a minimum at $d=280 \mathrm{pc}\left(\tilde{\chi}_{280}^{2}=0.97\right)$. The $90 \%$ confidence interval is enclosed in the dashed lines and covers the range $230<d<320 \mathrm{pc}$.

We fixed the model parameters (with the exception of the distance) to those evaluated for FeII and ran the photoexcitation code for SiII. As noted in the previous subsection, the SiII ground state column density for component IV (Table 2) should be interpreted with caution, since it is measured from a single absorption line, that is saturated. In a steady-state approximation, we would expect the ratio of the column density of SiII fine structure to ground levels to be $\sim 10$ times higher than the corresponding FeII ratio (see e.g., Prochaska et al. 2006). Thus, the SiII $\lambda 1526$ value that we found is unusual, since the ratio is 0.1 for FeII while the one for SiII is only 0.07. This means that we are overestimating the SiII ground state column density and we should consider our value as an upper limit to the real density. The result of the photoexcitation code for the SiII levels is shown in Fig. 5. The distance that we find is $d=800 \mathrm{pc}$, which accordingly should be considered as an upper limit consistent with the estimation for the FeII. We also note that for a steady-state approximation our SiII column densities would imply a distance from the GRB 3-5 times higher than that inferred from FeII. This is again consistent with the results of our time-dependent code. 


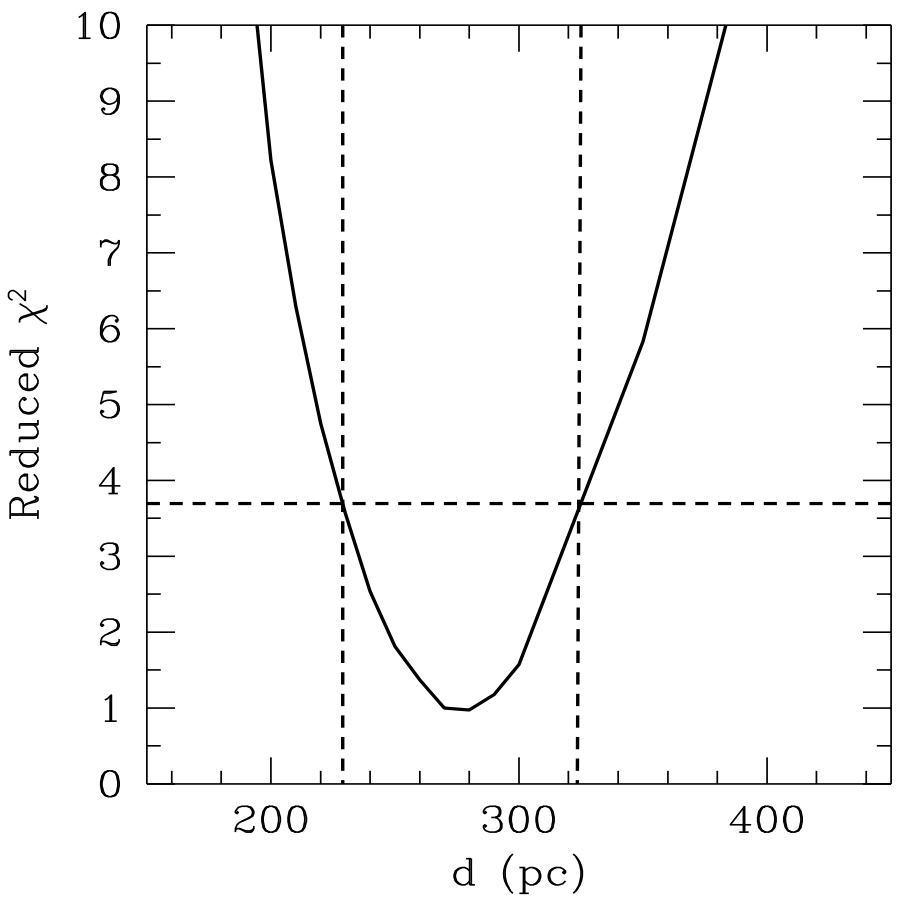

Fig. 4. The $\tilde{\chi}^{2}$ as a function of the distance of the absorber from the GRB. It defines the quality of the agreement between the observed FeII column densities and that predicted by our time-dependent photoexcitation code (see also Fig. 3). The $\tilde{\chi}^{2}$ has a minimum at $d=280 \mathrm{pc}$, and the $90 \%$ confidence distance range is enclosed by dashed lines.

To ensure self-consistency, we need to establish that, at the distance of $280 \mathrm{pc}$ as derived for component IV, FeII and MgI are not fully photoionized by the strong UV radiation of the burst. For this purpose, we performed a series of runs of the photoionization code by Perna \& Lazzati (2002), which accounts for the radiative-transfer of the radiation. We first simulated a medium in thermal equilibrium at a temperature of $\sim 10^{4} \mathrm{~K}$, and allowed the radiation from the burst, modeled by Eq. (1), to propagate through the medium for $40 \mathrm{~min}$ in the rest frame. We found that for absorber densities in the range of $1-10^{3} \mathrm{~cm}^{-3}$, the FeII column density is substantially unchanged after $40 \mathrm{~min}$. The column density of $\mathrm{MgI}$ is reduced by $\sim 7 \%$ for densities of $\sim 1 \mathrm{~cm}^{-3}$, by $\sim 3 \%$ for $\sim 10^{2} \mathrm{~cm}^{-3}$, and is unaltered for $\sim 10^{3} \mathrm{~cm}^{-3}$. We can thus safely conclude that there is a wide range of densities for which FeII and MgI are not substantially photoionized if the $\mathrm{GRB}$ is at $280 \mathrm{pc}$.

\section{The intervening systems}

The absorption lines produced by the gas belonging to the GRB host galaxy are the dominant features of the high resolution spectrum, but they are not the only ones. The signal-to-noise level of our spectrum allowed us to detect lines with equivalent widths as weak as $0.06 \AA$, at the $2 \sigma$ confidence level. A detailed analysis of the data found that at least three other absorbers are present along the line of sight to GRB 080330. The two higher redshift ones $(z=1.0179$ and $z=1.0166)$ show absorption only from the $\mathrm{MgII} \lambda 2796, \lambda 2803$ doublet, and exhibit a very simple line profile, that can be fitted by a single Voigt function. However, since they are only separated by $\sim 200 \mathrm{~km} \mathrm{~s}^{-1}$, they should be interpreted as a single, clumpy system (Fig. 6). The third absorber is rather complicated. The absorption lines detected for this system are the $\operatorname{MgII} \lambda 2796, \lambda 2803$ doublet and the $\mathrm{MgI} \lambda 2852$ line.

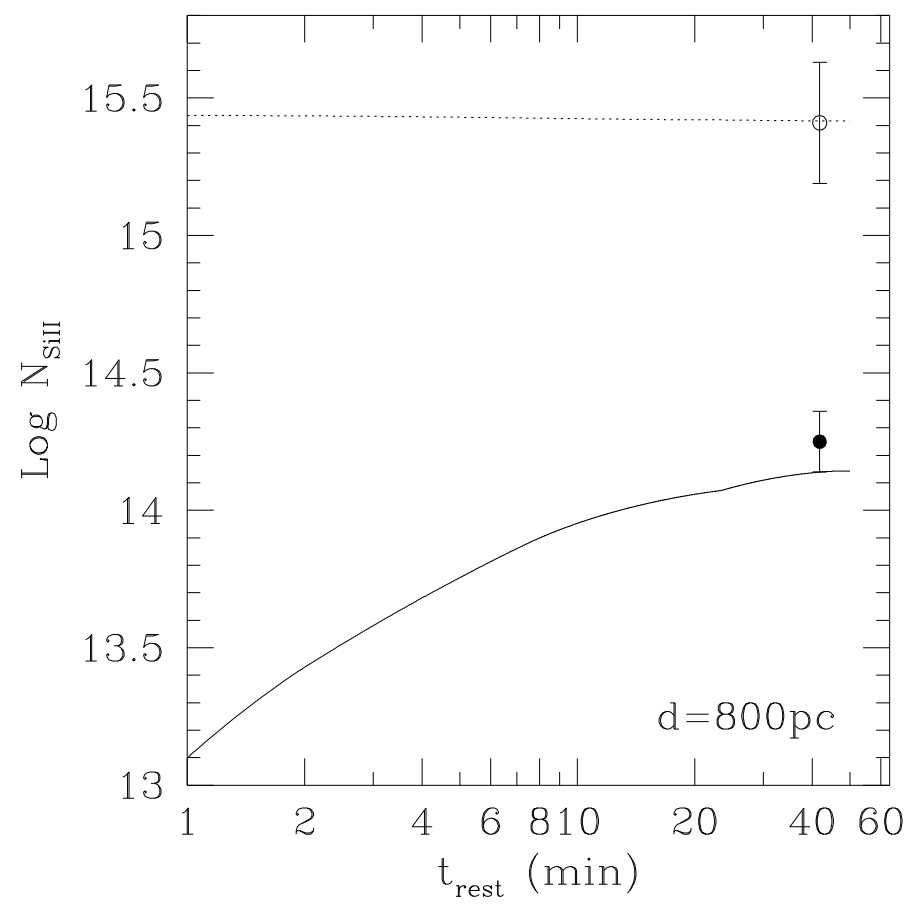

Fig. 5. The column densities for the ground level of SiII (open circle) and fine structure transition for component IV in the spectrum of GRB 080330. Column density predictions from our time-dependent photo-excitation code are also shown. They refer to the ground level (dotted line) and fine structure level (solid line) transitions, in the case of an absorber at $800 \mathrm{pc}$ from the GRB. However, this value should be considered as an upper limit (see text for details).

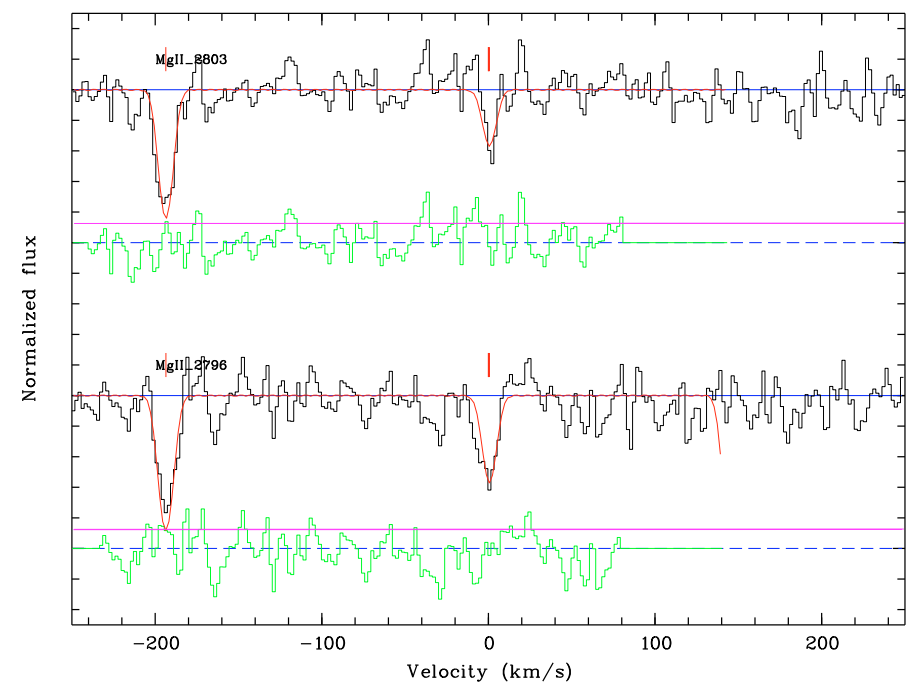

Fig. 6. The intervening system at $z=1.02$. MgII is the only ion observed in this system with its $\lambda 2796$ and $\lambda 2803$ doublet. Solid lines represent our best-fit model (see also Table 3). Bottom, solid lines mark the fit residuals.

This system has a complex structure and a single Voigt profile is not adequate to reproduce it. At least five different components are needed to obtain a good fit to the MgII data. The bluemost component is saturated, and so a reliable column density for the MgII cannot be obtained. However, this is the only component that exhibits MgI absorption at the $90 \%$ confidence level. This MgI component needs a more complex modeling to adequately follow its profile. This results in a further division into 


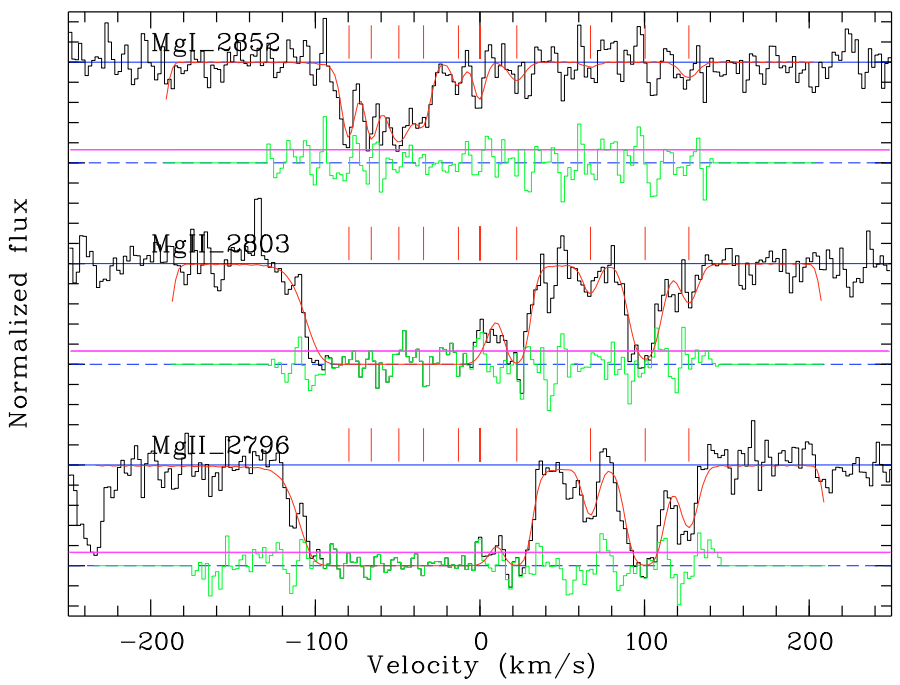

Fig. 7. The complex intervening system at $z=0.8213$. A fivecomponent model was required for the best-fit to the dataset. The neutral $\mathrm{MgI}$ and the ion $\mathrm{MgII}$ are the only observed absorbers, with the $\lambda 2852$ transition and $\lambda 2796-\lambda 2803$ doublet, respectively. MgI shows significant absorption in the bluemost component only, which needs a subcomponent modeling to obtain a good fit. MgII shows absorption in all components; the bluemost is saturated (see also Table 3). Bottom, solid lines mark the fit residuals.

Table 3. Absorption-line rest-frame equivalent widths and column densities for the intervening systems.

\begin{tabular}{lcc|ccc}
\hline \hline System & Species & Transition & $z$ & $E W \lambda 2796(\AA)$ & $N^{a}$ \\
\hline 1 & MgII & $\lambda 2796, \lambda 2803$ & 1.0179 & $0.06 \pm 0.03$ & $12.33 \pm 0.07$ \\
\hline 1 & MgII & $\lambda 2796, \lambda 2803$ & 1.0166 & $0.08 \pm 0.03$ & $13.47 \pm 0.19$ \\
\hline 2 & MgII & $\lambda 2796, \lambda 2803$ & 0.8222 & & $12.49 \pm 0.03$ \\
2 & MgII & $\lambda 2796, \lambda 2803$ & 0.8220 & & $13.34 \pm 0.03$ \\
2 & MgII & $\lambda 2796, \lambda 2803$ & 0.8219 & $1.70 \pm 0.04^{b}$ & $12.24 \pm 0.04$ \\
2 & MgII & $\lambda 2796, \lambda 2803$ & 0.8216 & & $14.04 \pm 0.09$ \\
2 & MgII & $\lambda 2796, \lambda 2803$ & 0.8213 & & Sat. \\
\hline 2 & MgI & $\lambda 2852$ & 0.8213 & $0.79 \pm 0.04$ & $13.97 \pm 0.15$ \\
\hline
\end{tabular}

${ }^{a}$ Column densities $N$ are logarithmic $\mathrm{cm}^{-2}$; Sat marks saturated components.

${ }^{b}$ The rest equivalenth width of the $\lambda 2796$ transition for the last two components only $(z=0.8213$ and 0.8216$)$ is $1.30 \pm 0.04$.

six subcomponents (Fig. 7). Taking into account the velocity spread of $\sim 260 \mathrm{~km} \mathrm{~s}^{-1}$, we consider this system as a single complex absorber. The redshift path analyzed for the search of MgII systems lies in the intervals $z=0.25-0.38, z=0.70-1.05$, and $z=1.08-1.51$.

Table 3 summarizes the rest-frame equivalent widths, column densities, and redshifts calculated for these intervening systems. Lines 1 and 2 refer to the first double system, while lines 3-7 refer to the more complex, low redshift one. We report only the total column density for the bluemost component of MgI (the corresponding $\mathrm{MgII}$ is saturated), and do not report the values of all the six subcomponents. The equivalent width is computed for this system as a whole.

\section{Conclusions}

We have presented high resolution $(R=40000$, corresponding to $7.5 \mathrm{~km} \mathrm{~s}^{-1}$ ) spectroscopy of the optical afterglow of the
Gamma Ray Burst GRB 080330, observed using UVES at the VLT $\sim 1.5 \mathrm{~h}$ after the trigger.

We confirm that the redshift of the host galaxy is $z=1.51$. The spectrum shows that the ISM of the GRB host galaxy is complex, with at least four components contributing to its main absorption system at $z=1.51$. These components are identified in this paper with progressive numbers of decreasing velocity values.

These absorption lines appear both as neutral metalabsorption lines (MgI) and low ionization lines (FeII, SiII, MnII, CrII, ZnII).

In addition, we detect strong SiII, NiII, and FeII excited transitions, but only in component IV (i.e., the bluemost component of the main absorption system). Since indirect UV pumping is likely to be the leading mechanism in exciting these features (Vreeswijk et al. 2007), we conclude that component IV is the one more affected by the GRB afterglow, meaning that is the closest to the GRB progenitor. This distance can be constrained by comparing the column densities of the ground and excited levels to those predicted by a time-dependent photoexcitation code for the hypothesis of indirect UV-pumping. This comparison has been carried out for FeII and SiII only; in fact, the column density of the NiII ground level could not be measured, because the corresponding absorption transitions fell in the gap between the blue and the red regions of the spectrum. We found that the absorbing gas of component IV is located at $\sim 280_{-50}^{+40} \mathrm{pc}$ from the GRB. To ensure self-consistency, we ran a photoexcitation code that confirms that there is a wide range of densities for which the FeII and MgI are not substantially photoionized if the GRB is at $280 \mathrm{pc}$. If this is the case, since the abundance ratio $[\mathrm{Cr} / \mathrm{Zn}]=-0.2$ (comparable with DLAs, see Prochaska et al. 2006), while $[\mathrm{Fe} / \mathrm{Zn}]=-0.9$ (among the lowest value for DLAs), the absorber appears to be very dusty.

Although the distance between the GRB and the absorber is smaller than the value found from a similar analysis in other GRBs (1.7 kpc for GRB 060418, Vreeswijk et al. 2007; and 2-6 kpc for GRB 080319B, D'Elia et al. 2009), it nevertheless confirms that the power of a GRB affects a region of gas that is at least a few hundreds of $\mathrm{pc}$ in size.

The host galaxy of the GRB is not the only absorber located along the line of sight to the afterglow. At least two more intervening systems between $z=1.02$ and $z=0.82$ have been identified. The system at the highest redshift can be modeled simply with a double Voigt profile and exhibits absorption only in the MgII $\lambda 2796, \lambda 2803$ doublet. On the other hand, the second is complex, featuring at least five different components; given the limited velocity interval however, we assume this to be one single, clumpy structure along the line of sight. This system exhibits only MgII $\lambda 2796, \lambda 2803$ doublet and MgI $\lambda 2852$ line absorption.

One of these systems has a rest-frame equivalent width larger than $1 \AA$. This follows the trend that on average one strong intervening system is found per afterglow, as has been noted in studies that have detected excess of strong MgII absorbers along GRB sightlines compared to quasars.

Acknowledgements. We thank an anonymous referee for several helpful comments. We acknowledge support from ASI/INAF contracts ASI/I/R/039/04 and ASI/I/R/023/05/0.

\section{References}

Ballester, P., Modigliani, A., Boitquin, O., et al. 2000, ESO Messenger, 101, 31 Bloom, J. S., \& Starr, D. L. 2008, GCN Circ, 7542 
Boettcher, M., Fryer, C. L., \& Dermer, C. D. 2002, ApJ, 567, 441

Chen, H. W., Prochaska, J. X., Bloom, J. S., \& Thompson, I. B. 2005, ApJ, 634, $25 \mathrm{~L}$

Clemens, C. 2008, GCN Circ, 7545

Cobb, B. E. 2008, GCN Circ, 7553

Cucchiara, A., et al. 2008, GCN Circ, 7547

Dekker, H., D’Odorico, S., Kaufer, A., Delabre, B., \& Kotzlowski, H. 2000, SPIE, 4008, 534D

D’Elia, V., Fiore, F., Meurs, E. J. A., et al. 2007, A\&A, 467, 629

D'Elia, V., Fiore, F., Perna, R., et al. 2009, ApJ, 694, 332

Draine, B. T., \& Hao, L. 2002, ApJ, 569, 780

Fiore, F., D’Elia, V., Lazzati, D., et al. 2005, ApJ, 624, 853

Golenetskii, S., Aptekar, R., Mazets, E., et al. 2008, GCN Circ, 7548

Guidorzi, C., Clemens, C., Kobayashi, S., et al. 2009, A\&A, in preparation

Klotz, A., Boer, M., \& Atteia, J. L. 2008, GCN Circ, 7536

Malesani, D., Fynbo, J. P. U., Jakobsson, P., Vreeswijk, P. M., \& Niemi, S.-M. 2008, GCN Circ, 7544

Mao, J., Beumgarther, W. H.; Burrows, D. N.; et al. 2008, GCN Circ, 7537

Mirabal, N., Halpern, J. P., Kulkarni, S. R., et al. 2002, ApJ, 578, 818

Penprase, B. E., Berger, E., Fox, D. B., et al. 2006, ApJ, 646, 358
Perna, R., \& Lazzati, D. 2002, ApJ, 580, 261

Perna, R., \& Loeb, A. 1998, ApJ, 501, 467

Perna, R., Lazzati, D., \& Fiore, F. 2003, ApJ, 585, 775

Piranomonte, S., Ward, P. A., Fiore, F., et al. 2008, A\&A, 492, 775

Prochaska, J. X., Gawiser, E., Wolfe, A. M., Castro, S., \& Djorgovski, S. G. 2003, ApJ, 595, L9

Prochaska, J. X., Chen, H. W., \& Bloom, J. S. 2006, ApJ, 648, 95

Prochaska J. X., Chen, H. W., Dessauges-Zavadsky, M., \& Bloom, J. S. 2007, ApJ, 666, 267

Prochter, G. E., Prochaska, J. X., Chen, H.-W., et al. 2006, ApJ, 648, L930

Savaglio, S., Glazebrook, K., Crampton, D., et al. 2004, ApJ, 602, 51

Savaglio, S., Glazebrook, K., Le Borgne, D., et al. 2005, ApJ, 635, 260

Schaefer, B. E., \& Guver, T. 2008, GCN Circ, 7538

Steidel, C. C., Adelberger, K. L., Giavalisco, M., Dickinson, M., \& Pettini, M. 1999, ApJ, 519, 1

Sudilovsky, V., Savaglio, S., Vreeswijk, P, et al. 2007, ApJ, 669, 741

Tejos, N., Lopez, S., Prochaska, J. X., Chen, H.-W., \& Dessauges-Zavadsky, M. 2007, ApJ, 671, 622

van Marle, A. J., Langer, N., \& Garcia-Segura, G 2005, A\&A, 444, 837

Vreeswijk, P. M., Ledoux, C., Smette, A., et al. 2007, A\&A, 468, 83 